\title{
RECOMBINATION KINETICS IN LOW DIMENSIONS
}

Panos ARGYRAKIS

Department of Physics, University of Thessaloniki, GR-54006 Thessaloniki, Greece

and

Raou1 KOPELMAN

Department of Chemistry, University of Michigan, Ann Arbor, MI 48109, U.S.A.

Computer simulations predict very anomalous delayed luminescence decays for low-dimensional materials. Classical kinetics theory fails because excitons, electrons and holes have no convection. The mode of stirring plays a very important role in the rate of reactions.

The laws of chemical reaction kinetics are customarily used for recombination kinetics. This implies time-independent rate constants (K). For instance, for the triplet exciton fusion process $A+A \rightarrow A_{2}$, leading to delayed fluorescence, one gets

$$
F=K P^{2} \quad K=\text { constant }
$$

where $F$ designates the delayed fluorescence and $P$ the phosphorescence. This relation has been questioned $^{1}$ in experiments with isotopic mixed naphthalene single crystals at low temperatures. Further corroboration is given by computer simulations, where a chemical reaction is simulated us ing random walk models for the reacting species, ${ }^{2}$ showing that $k=k(t)$ goes down monotonically with time for low-dimensional reaction spaces. This is the case when the reacting-particle motion is diffusion-limited. We monitor the diffusion limited process of reactions by introducing the idea of stirring the reactants during the course of reaction. Stirring has an effect of breaking up the nonrandom spatial and temporal distri-butions caused by the reaction. While it is easily

Supported by NSF Grant DMR-8303919 seen that constant stirring produces the classical result, the effects of partial stirring are not yet clear, since partial stirring is characterized, in part, by diffusion and, in part, by classical behavior. This is investigated in the current work.

In Fig. 1 we compare the $A+A \rightarrow A_{2} \uparrow$ with the $A+B \rightarrow A B \uparrow$ reactions for the $1-d i m$ topology. We observe that trends of the $A+A$ reaction are paralleled in the $A+B$ reaction: The nonconstant $K=K(t)$ in the diffusion-limited case and the constant $k$ in the well-stirred case.

In Fig. 2 we investigate the effect of stirring on the reaction rate. There is no conventional method of such stirring available and the consequences for the rate coefficient are not immediately obvious. We tried several models, covering the whole range from no stirring (a), to the case of a well-stirred reaction (b), where all particles are stirred constantly, i.e. at each time-step. Intermediate mechanisms include: (I) Stirring of all particles once every 400 steps (c); (II) stirring of a fraction (1\%) of particles only (d), randomly chosen; and, finally, (III) the case of local stirring (e) where every particle is constantly stirred, 


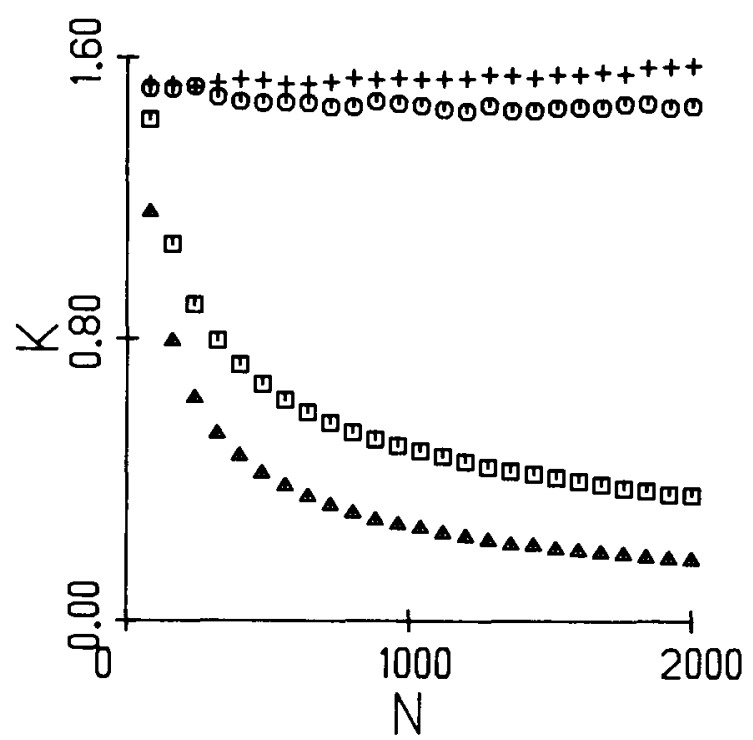

FIGURE 1

1-dim lattice, rate coefficient as a function of time for the following cases: (a) $\boldsymbol{U}: A+A$ reaction, diffusion-limited; (b) $O: A+A$ reaction, well-stirred; (c) $\Delta: A+B$ reaction, diffusion-limited; (d) +: A + B reaction, wel1stirred. The initial density $\rho=0.05$ (for the $A+A$ reaction) and $\rho=0.05$ f $8 \mathrm{r}$ each of $A$ and $B$ (for the $A+B$ reaclion).

but stays in its close neighborhood, as for example within a maximum range of 5 sites away from its original position. We observe for the $A+B$, two-dim reaction of this figure that model (d) is almost similar to the diffusionlimited case (a) while model (e) is similar to the well-stirred one (b). These observations verify the original assumption of having a nonrandom distribution of reactants during the course of the reaction. A small spatial perturbation caused by randomizing over a range of only 5 sites away is enough to totally change the character of the reaction (model e), while a large spatial perturbation on a limited number of particles ( $1 \%$ in model d) is not sufficient to cause such change. Stirring at long intervals (every 400 steps, model c) shows that

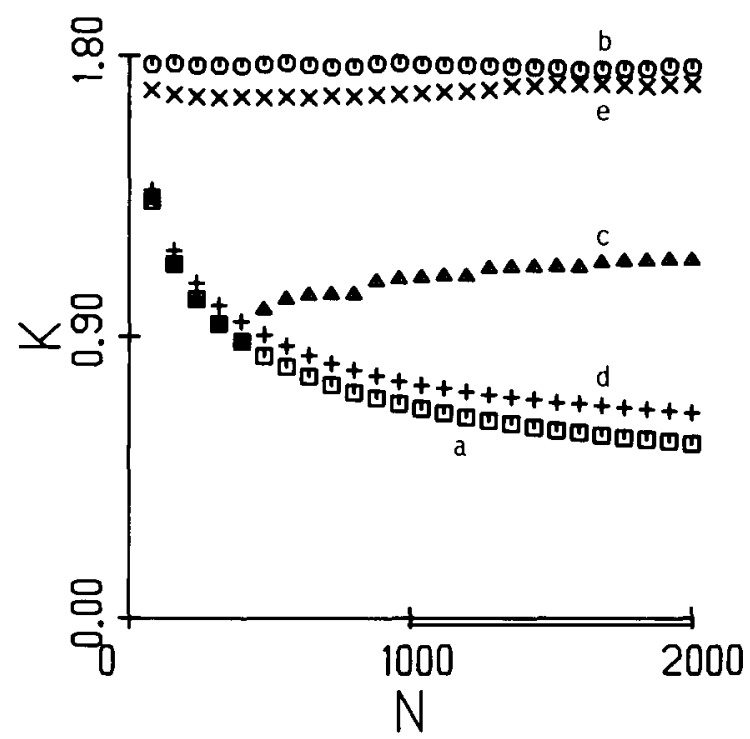

FIGURE 2

2-dim, A + B reactions. Rate coefficient as a function of time for different modes of stirring (see text). Initial density $\rho_{0}=0.05$ for each of $A$ and $B$.

for the time domain of no stirring the reaction parallels the diffusion-limited case, as expected, while after the commencement of stirring the correlation is lost and $k$ increases momentarily. Thus it is shown that the rate coefficient of a reaction depends on the mechanism of stirring used. Experimentally, partial stirring of excitons may be achieved, for example, via thermalization from the guest system to the host band. 4

\section{REFERENCES}

1. P. W. KTymko and R. Kopelman, J. Lumin. $24 / 25$ (1981) 457 .

2. P. Argyrakis and R. Kopelman, J. Phys. Chem. 91 (1987) 2699.

3. R. Kope Iman, J. Hoshen, J. S. Newhouse and P. Argyrakis, J. Stat. Phys. 30 (1983) 355.

4. E. M. Monberg and R. Kopelman, Mol. Cryst. Liq. Cryst. 57 (1980) 271. 